\title{
Allegra Transcatheter Heart Valve inside a Degenerated Sutureless Aortic Bioprosthesis
} \author{
der Fulda, Germany
Abstract
Keywords
- heart valve
- transapical
- reoperation
- aortic valve and root
- cardiologyl cardiologist \\ - percutaneous
}

Maximilian Vondran ${ }^{1,2}$

Bernd $\mathrm{Abt}^{3}$ Holger $\mathrm{Nef}^{3,4}$

1 Department of Cardiac and Thoracic Vascular Surgery, University Hospital Giessen and Marburg, Campus Marburg, Marburg, Germany

2 Department of Cardiac and Vascular Surgery, Herz-KreislaufZentrum, Rotenburg an der Fulda, Germany

3 Department of Cardiology, Herz-Kreislauf-Zentrum, Rotenburg an

${ }^{4}$ Department of Cardiology, University Hospital Giessen and

Marburg, Campus Giessen, Giessen, Germany

Thorac Cardiovasc Surg Rep 2021;10:e1-e5.

\author{
Ardawan J. Rastan ${ }^{1,2}$
}

\begin{abstract}
Address for correspondence Maximilian Vondran, MD, Department of Cardiac and Thoracic Vascular Surgery, Philipps-University Hospital Marburg, Baldingerstraße, 35043 Marburg, Germany (e-mail: m.vondran@hkz-rotenburg.de).
\end{abstract}

\section{Introduction}

In the therapy of degenerated surgical aortic valve bioprostheses (SAVs), transcatheter aortic valve-in-valve implantation (VIV) is increasingly being used successfully. ${ }^{1,2}$ However, the sutureless SAV (Perceval S; LivaNova, London, United Kingdom), as a representative of the latest generation of SAVs, has become established in the standard surgical treatment of severe aortic valve stenosis (AS) and has gained popularity worldwide due to its good hemodynamic outcome and short implantation time. ${ }^{3}$ However, these SAVs also degenerate and are, therefore potential candidates for VIV procedures. The new, self-expanding Allegra transcatheter heart valve (THV) (New Valve Technology, Hechingen, Germany) has recently been shown its safety and feasibility for VIV in patients with a small degenerated SAV, producing excellent hemodynamic results. ${ }^{4}$ Here we describe the first successful VIV with the Allegra THV into a degenerated sutureless SAV.

\section{Case Presentation}

An 85-year-old female patient (weight: $84.3 \mathrm{~kg}$, height: $163 \mathrm{~cm}$ ) presented at our institution with recurrent, worsening dyspnea with light physical activity. Three years previously, she had undergone surgical aortic valve replacement with the Perceval S (size M) due to severe AS via a partial upper mini-sternotomy. On admission, the transesophageal and transthoracic echocardiography showed severe stenosis of the sutureless SAV with a maximum/mean transvalvular gradient of $79 / 55 \mathrm{~mm} \mathrm{Hg}$, an effective orifice area of $0.6 \mathrm{~cm}^{2}$, an internal diameter of the $S A V$ of $17 \mathrm{~mm}$, a received

September 10, 2020 accepted after revision October 15, 2020
DOI https://doi.org/ 10.1055/s-0040-1721483. ISSN 2194-7635.

\footnotetext{
(C) 2021. The Author(s).

This is an open access article published by Thieme under the terms of the Creative Commons Attribution-NonDerivative-NonCommercial-License, permitting copying and reproduction so long as the original work is given appropriate credit. Contents may not be used for commercial purposes, or adapted, remixed, transformed or built upon. (https://creativecommons.org/ licenses/by-nc-nd/4.0/) Georg Thieme Verlag KG, Rüdigerstraße 14, 70469 Stuttgart, Germany
} 

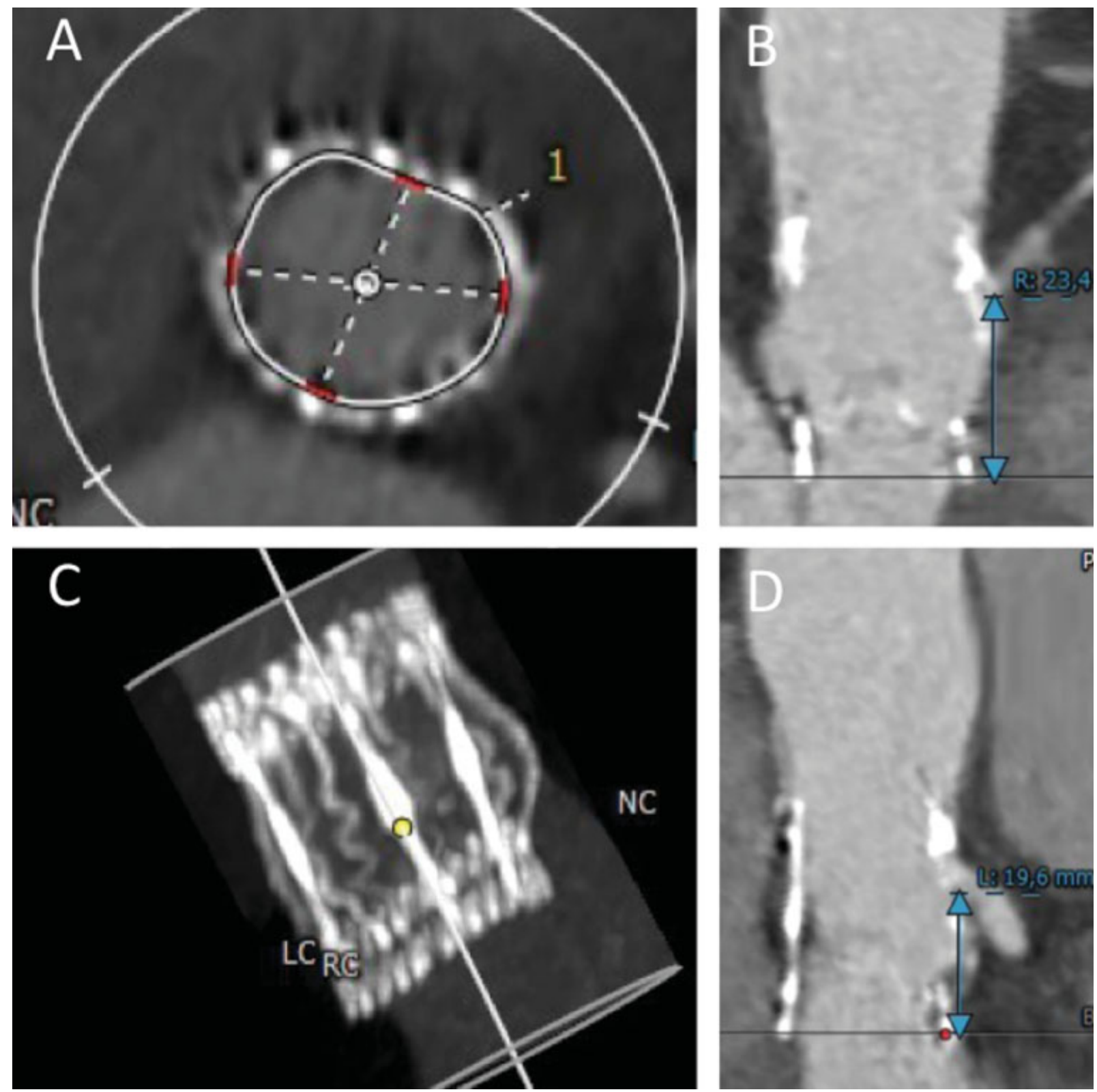

Fig. 1 Multilayer spiral computed tomography. Annulus plane of the sutureless surgical aortic valve bioprostheses (A), right coronary (RC) ostium height (B), stent frame of the surgical sutureless aortic valve bioprosthesis (NC = non-coronary) (C), left coronary (LC) ostium height (D).

mild aortic regurgitation without any paravalvular leakage (PVL), and severe tricuspid valve regurgitation. The left ventricular ejection fraction was $55 \%$, but the right heart function was impaired (tricuspid annular plane systolic excursion of $15 \mathrm{~mm}$ ). A multislice spiral computed tomographic (CT) scan showed a typically shaped aortic root and ascending aorta without any calcification of the aortic valvular cusps. The stent frame of the sutureless SAV was not dislocated but was slightly oval shaped (- Figs. 1 and 2). Due to the age and increased surgical risk EuroSCORE (European System for Cardiac Operative Risk Evaluation) II: 6.21\% and Society of Thoracic Surgeons Score: $11.9 \%$ ), the patient was considered to be a candidate for a transcatheter VIV by the heart team. According to the manufacturer, the inner diameter of the size-M Perceval S was between 19.5 and $21.0 \mathrm{~mm}$. However, CT scan measurements were made to determine the true internal diameter of the sutureless SAV (annulus area: $303 \mathrm{~mm}^{2}$, annulus perimeter: $62.4 \mathrm{~mm}$, and effective annulus diameter: $19.3 \mathrm{~mm}$; - Fig. 1A).

Due to the implanted sutureless SAV with a very small effective, slightly oval-shaped annulus, and the preprocedural high transvalvular gradient, we needed a THV in this patient that could lead to an excellent hemodynamic result despite the unfavorable preinterventional conditions. Therefore, we decided to implant a self-expandable, supra-annular THV. Based on the promising data on its use in VIV therapy inside SAVs with small true inner diameters, ${ }^{4}$ we chose the $23-\mathrm{mm}$ Allegra THV in this patient.

We performed the VIV procedure in a standard manner (-Video 1) and used a $20 \mathrm{~mm} \times 40 \mathrm{~mm}$ noncompliant balloon for predilatation (-Fig. $\mathbf{3 B}$ ) to modulate the annulus 


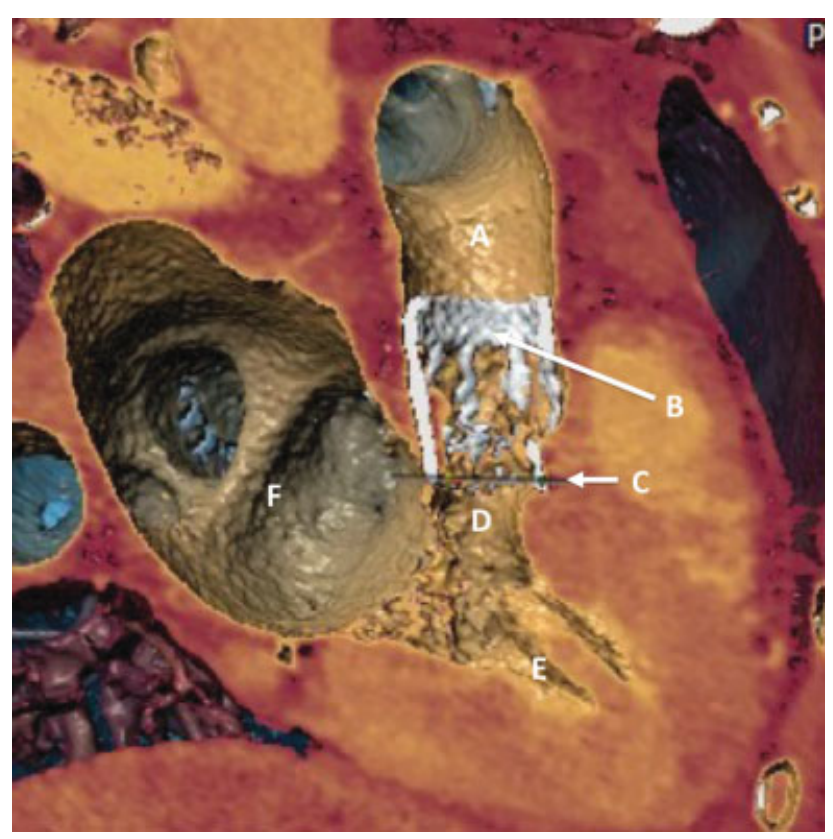

Fig. 2 Multilayer spiral computed tomography. Volume rendering of the aortic root with the sutureless SAV inside. Ascending aorta (A), stent frame of the surgical sutureless aortic valve bioprosthesis (B), aortic annulus (C), left ventricular outflow tract (D), left ventricle (E), and left atrium (F).

of the SAV more round. The Allegra THV was first positioned optimally within the annulus of the sutureless SAV and the "permaflow mode" was activated (-Fig. 3C). Subsequently, the lower part of the device was freed by "tip release" (-Fig. 3D). No postdilatation was needed in view of the excellent hemodynamic results with an invasive transvalvular gradient of $5 \mathrm{mmHg}$. Based on The Valve Academic Research Consortium II criteria, no major adverse event occurred during the hospital stay. Hypostatic pneumonia was treated with systemic antibiotics, and a puncture evacuated pleural effusion on the right side. The patient was discharged 9 days after the procedure to a rehabilitation center with a maximum/mean transvalvular pressure gradient measured by transthoracic echocardiography of 19/9 mm Hg and no apparent PVL.

\section{Video 1}

Fluoroscopy of the transcatheter aortic valve-in-valve implantation procedure. Online content including video sequences viewable at: https://www.thieme-connect. com/products/ejournals/html/10.1055/s-00401721483.

\section{Discussion}

Due to the natural degeneration process of SAVs, the frequency of VIV in sutureless SAV will increase in the coming years. Considering the very promising hemodynamic results of the Allegra THV, ${ }^{4}$ it is an excellent candidate for use in VIV procedures in SAVs with a small true internal diameter. However, it has never been used in degenerated sutureless SAV.

A strong argument for using self-expanding THVs in VIV procedures is less-structural valve deterioration in the long-term compared with balloon-expandable valves in the therapy of native AS. ${ }^{5}$ This difference is likely to become more critical as the native aortic valve annulus diameter or the inner diameter of a degenerated SAV decreases over time. ${ }^{4}$ Moreover, the self-expanding THV is superior in the short-term hemodynamic outcome after VIV therapy. ${ }^{6}$ The benefit of the self-expanding THV is that they have fewer problems with incomplete deflation or distortion within a degenerated SAV. The supra-annular valve design is less vulnerable and usually results in a larger effective orifice area and a lower transvalvular gradient, almost regardless of a small and/or asymmetric annulus. Despite their excellent hemodynamic long-term outcome, above-mentioned positive features, and the recapture ability of some devices, they are used in less than one-third of VIV in latest-generation SAVs. ${ }^{7}$ Unfortunately, with some self-expanding THVs, accessing the coronary arteries after VIV can be difficult. However, due to a sophisticated closed-cell, diamond-shaped configuration of the nitinol stent frame with a variable cell size distribution, the Allegra THV allows easy access for potential percutaneous coronary intervention at a later stage. With the implantation of the popular self-expanding Evolut R THV (Medtronic Inc., Minneapolis, Minnesota, United States) in our case, postinterventional access to the coronary arteries would have been nearly impossible. Also, the stent frame configuration of the Allegra THV leads to improved coronary perfusion and very low postinterventional transvalvular gradients, even after VIV. ${ }^{4}$ In our case, we were able to reduce the maximum/mean transvalvular pressure gradient from $79 / 55 \mathrm{~mm} \mathrm{Hg}$ to 19 / $9 \mathrm{~mm} \mathrm{Hg}$. Furthermore, different levels of radial force enhance a safe anchoring of the Allegra within the aortic annulus. The six radiopaque gold markers placed at the valve plane level to indicate the bottom part of the semilunar valve assist the operator in positioning this THV corrected, especially during VIV. The prosthesis's ventricular inflow section is covered by a bovine pericardial sealing skirt to mitigate paravalvular prosthetic regurgitation. Unfortunately, no direct comparisons of the performance in VIV of the Allegra and other self-expanding THVs have been made. However, the Evolut $R$ and the Portico (St. Jude Medical, Inc., St. Paul, Minnesota, United States) were compared in a matched analysis based on the VIVID registry; here, the Evolut $\mathrm{R}(n=108)$ was superior to the Portico $(n=54)$ in terms of postinterventional effective orifice area and mean gradient (1.67 vs. $1.31 \mathrm{~cm}^{2} ; p=0.001$ and $14 \pm 7.5$ vs. $17 \pm 7.5 \mathrm{~mm} \mathrm{Hg}$; $p=0.02$, respectively). ${ }^{8}$ In the multicentric VIVAL trial $(n=30)$ of the Allegra in VIV procedures, these parameters (effective orifice area: $1.40 \pm 0.52 \mathrm{~cm}^{2}$, mean gradient: $14.8 \pm 6.5 \mathrm{~mm} \mathrm{Hg}^{4}$ are comparable to those for the Evolut $\mathrm{R}$ from the VIVID registry. In our case, the good 


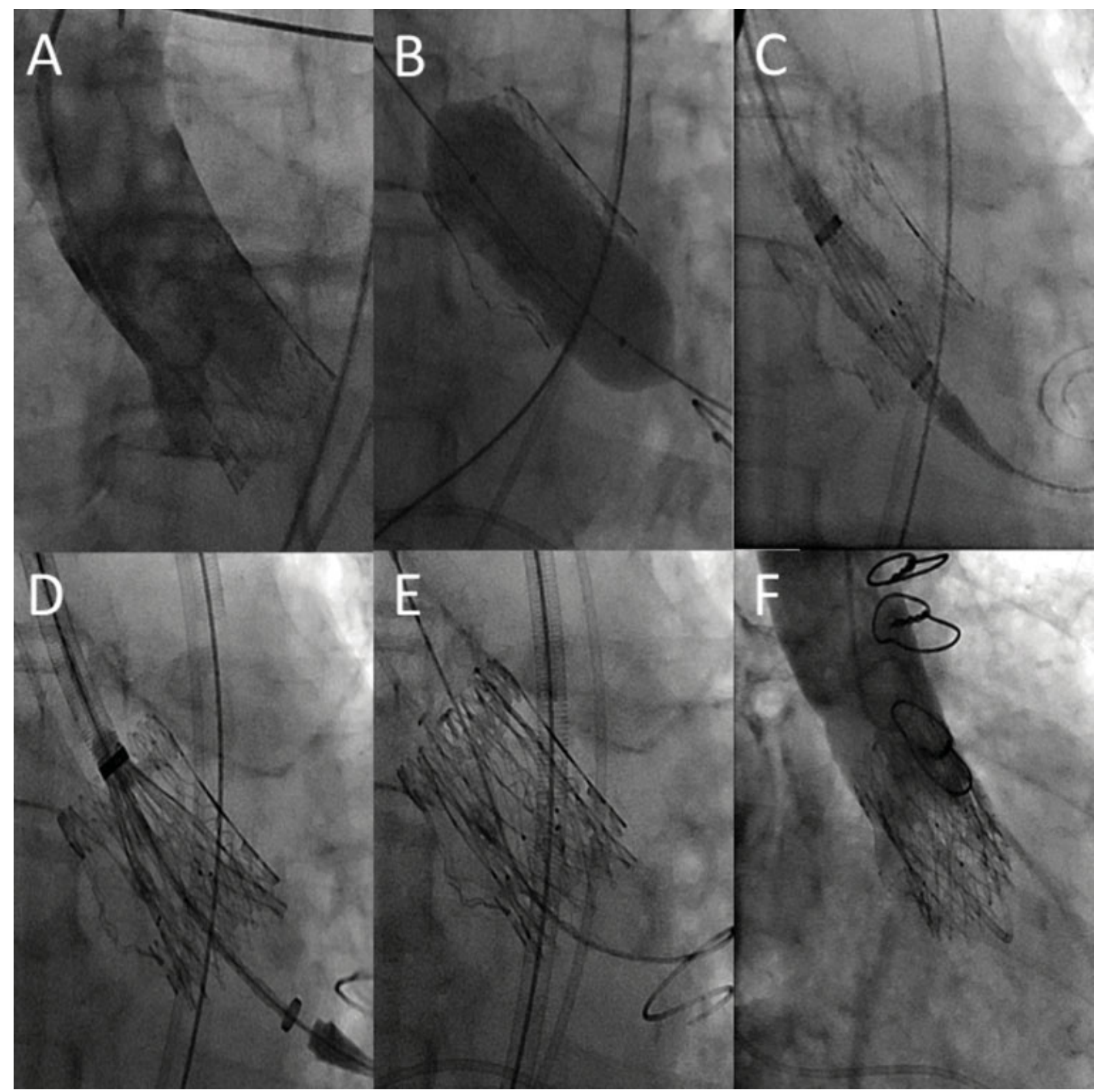

Fig. 3 Fluoroscopy of the transcatheter aortic valve-in-valve implantation procedure. Preimplantation aortography (A), predilatation valvuloplasty (B), permaflow mode (C), tip-release (D); final result (E), and postimplantation aortography (F).

hemodynamic properties of the Allegra were confirmed, even in the small annulus of the degenerated sutureless SAV.

\section{Conclusion}

A VIV procedure using the Allegra THV into the Perceval S sutureless SAV is feasible with an excellent hemodynamic result.

\section{Funding \\ None.}

\section{Conflict of Interest}

None declared.

\section{References}

1 Deeb GM, Chetcuti SJ, Reardon MJ, , et al. 1-year results in patients undergoing transcatheter aortic valve replacement with failed surgical bioprostheses. JACC Cardiovasc Interv 2017;10(10):1034-1044

2 Webb JG, Mack MJ, White JM, , et al. Transcatheter aortic valve implantation within degenerated aortic surgical bioprostheses: Partner 2 valve-in-valve registry. J Am Coll Cardiol 2017;69(18): 2253-2262

3 Powell R, Pelletier MP, Chu MWA, Bouchard D, Melvin KN, Adams C. The perceval sutureless aortic valve: review of outcomes, complications, and future direction. Innovations (Phila) 2017; 12(03):155-173

4 Schäfer U, Butter C, Landt M, , et al. Thirty-day outcomes of a novel transcatheter heart valve to treat degenerated surgical valves: The vivall multicentre, single-arm, pilot study. EuroIntervention 2019;15(09):e757-e763

5 Abdel-Wahab M, Landt M, Neumann F-J, , et al; CHOICE Investigators. 5-year outcomes after tavr with balloon-expandable 
versus self-expanding valves: Results from the choice randomized clinical trial. JACC Cardiovasc Interv 2020;13(09):1071-1082

6 Ochiai T, Yoon SH, Sharma R, , et al. Outcomes of self-expanding vs. Balloon-expandable transcatheter heart valves for the treatment of degenerated aortic surgical bioprostheses- a propensity score-matched comparison. Circ J 2018;82(10):2655-2662

7 Landes U, Dvir D, Schoels W, , et al. Transcatheter aortic valve-invalve implantation in degenerative rapid deployment bioprostheses. EuroIntervention 2019;15(01):37-43
8 Alnasser S, Cheema AN, Simonato M, , et al; Valve-in-Valve International Data Registry Investigators. Matched comparison of self-expanding transcatheter heart valves for the treatment of failed aortic surgical bioprosthesis: Insights from the valve-invalve international data registry (vivid). Circ Cardiovasc Interv 2017;10(04):e004392 